\title{
Contenidos de cariología impartidos por escuelas dentales chilenas: Estudio transversal.
}

\section{Contents of cariology taught by chilean dental schools: Cross-sectional study.}

\author{
Vicente Aránguiz-Freyhofer ${ }^{1 *}$, María Loreto Marró-Freitte ${ }^{1}$, \\ Valeria Ramírez-Lobos ${ }^{1}$, Gustavo Moncada-Cortes ${ }^{1}$.
}

\author{
1. Facultad de Odontología, Universidad de los \\ Andes, Chile. \\ * Correspondencia Autor: Vicente Aranguiz | \\ Dirección: El Director 6000 Of. 209, Las Condes \\ Santiago | Teléfono: +562 25017960 | E-mail: \\ vicente.aranguiz@gmail.com \\ Trabajo recibido el 16/06/2018. \\ Aprobado para su publicación el 05/07/2018
}

\begin{abstract}
RESUMEN
Objetivo: determinar los contenidos impartidos en cariología, su aplicación clínica y metodología educativa empleada, en las escuelas dentales chilenas de pregrado. Método: Se efectuó un estudio de corte transversal, en una muestra por conveniencia de docentes de cariología y/o asignaturas relacionadas con cariología en 23 de 29 escuelas de odontología chilenas (año 2015), n=13 acreditadas ante la Comisión Nacional de Acreditación y $n=10$ de 16 no acreditadas. Se envió un cuestionario de 55 preguntas por vía electrónica, basado en los dominios propuestos por la Asociación para la Educación Dental Europea. El cuestionario fue sometido al análisis y evaluación previa de contenido y lenguaje por un comité de expertos, los resultados fueron tabulados y analizados utilizando STATA v14.2. Resultados: La tasa de respuesta del cuestionario fue de $86,95 \%$. En promedio el $70 \%$ de los contenidos encuestados son impartidos, de ellos, el $65 \%$ se impartieron en clases teóricas, el $14 \%$ en práctica de laboratorio y se realizó aplicación clínica en el $24 \%$ de los contenidos impartidos. Conclusiones: La actual enseñanza de la cariología en las escuelas dentales chilenas es alta en contenidos teóricos, los que mayoritariamente no se imparten a través de práctica de laboratorio y la aplicación clínica de los contenidos desarrollados es escasa. PALABRAS CLAVE

Caries; Cariología; Educación en cariología; Enseñanza cariología.
\end{abstract}

Rev. Clin. Periodoncia Implantol. Rehabil. Oral Vol. 12(1); 31-36, 2019.

\begin{abstract}
The objective was to determine the current contents of cariology taught, its clinical application and educational methodology used in Chilean undergraduate dental schools. Materials and Method: A cross-sectional study was carried out in a convenience sample of cariology teachers and/or teachers of cariology-related subjects belonging to 23 of the 29 Chilean Dental Schools (year 2015), including $n=13$ out of 13 schools accredited by the National Accreditation Commission, and $n=10$ out of 16 non-accredited schools. A questionnaire of 55 questions was sent electronically, based on the domains proposed by the Association for European Dental Education (ADEE). The survey was subjected to prior analysis and evaluation of content and language by a panel of experts, the results were tabulated and analyzed using STATA v14.2. Results: The response rate o was $86.95 \%$. On average, $70 \%$ of the contents surveyed are taught, of which $65 \%$ were taught in didactic classes, $14 \%$ were in laboratory practice and $24 \%$ were taught in the clinical setting. Conclusions: The current teaching of cariology in chilean dental schools includes a large number of subjects taught mainly in didactic settings, with less in the laboratory practice, while the clinical application of the contents developed is scarce.

\section{KEY WORDS}

Caries; Cariology; Cariology education; Cariology teaching.
\end{abstract}

Rev. Clin. Periodoncia Implantol. Rehabil. Oral Vol. 12(1); 31-36, 2019.

\section{INTRODUCCIÓN}

A pesar de los reportes de reducción de la prevalencia y severidad, la caries dental continúa siendo un problema de salud pública en la mayoría de los países ${ }^{(1,2)}$, impactando significativamente la calidad de vida de las personas a todas las edades ${ }^{(3)}$. Adicionalmente, es la enfermedad crónica de mayor prevalencia en la especie humana y lidera el grupo de las cuatro enfermedades crónicas de más alto costo de tratamiento(4). En Chile, casi el $100 \%$ de la población adulta ha experimentado la enfermedad caries dental(5), similar a lo observado en otro país sudamericano como Brasil, con especial vulnerabilidad en los niveles socioeconómicos bajos y medios bajos ${ }^{(6)}$. 
En educación, la evaluación y constante adaptación de los contenidos son pre requisito para privilegiar las necesidades de los pacientes e incorporar nuevos contenidos, técnicas o procedimientos durante la enseñanza de la cariología ${ }^{(7)}$, implementando en la estructura curricular, los conocimientos de la etiopatogenia, prevención y tratamiento de la caries dental, tanto como los relacionados con otros desórdenes de los tejidos dentales, que responda a la necesidad de ofrecer educación pertinente con el estado actual de la enfermedad ${ }^{(8)}$. Varias propuestas han sido desarrolladas en el mundo en la enseñanza de la cariología. El currículo de Cariología en Europa, pareciera ser adecuadamente estructurado, mostrar buenos resultados y preferentemente enfocado a las políticas de control de la enfermedad basado en los acuerdos de Bologna, esta reformulación de la educación en odontología, ha requerido el compromiso del desarrollo del currículo único de contenidos, diseñado para todas las escuelas de los 48 países miembros ${ }^{(8,9)}$. Estudios sobre la educación en cariología en Japón ${ }^{(10)}$ y en Estados Unidos ${ }^{(11,12)}$ muestran que sus contenidos no están completamente alcanzados durante el pregrado y son de diversa profundidad entre las escuelas dentales. En relación a Latinoamérica, se observa que la enseñanza de la cariología está incluida en los currículos, con diferentes cargas académicas, detectándose ausencias y deficiencias de contenido entre las escuelas dentales ${ }^{(13)}$. El único estudio previo, sobre la educación en Cariología en las escuelas dentales chilenas muestra combinación de contenidos tradicionales y modernos, lográndose la implementación apropiada en la mitad de las escuelas dentales, sin alineación de los contenidos teóricos y el manejo clínico enseñados, presentándose acompañada de baja adopción de tratamientos no invasivos ${ }^{(14)}$. En general, la educación de pregrado en cariología presenta amplia gama de prácticas de enseñanza, siendo ineludible mejorar la alineación y criterios usados para calibrar a profesores y examinadores ${ }^{(15)}$.

En este contexto, en que la caries dental presenta significativo impacto social, económico y sanitario en la población chilena, se establece la necesidad de evaluar los contenidos educativos que imparten las escuelas dentales. Por este motivo, el objetivo del presente estudio fue determinar los actuales contenidos de cariología, que se imparten, su aplicación clínica y metodología educativa empleada en las escuelas dentales chilenas de pregrado.

\section{MATERIAL Y MÉTODO}

Se realizó un estudio de corte transversal, cuya estrategia fue el análisis de un cuestionario sobre los contenidos de cariología impartidos y su aplicación clínica, durante el proceso de enseñanza de la odontología en las Escuelas Dentales Chilenas de Pregrado (EDCP).

La muestra fue por conveniencia de acuerdo con los siguientes criterios de inclusión, A: Escuelas acreditadas ante la CNA (Comisión Nacional de Acreditación) que imparten odontología en Chile ( $n=13$ de 13) y b: Escuelas no acreditadas $n=10$ de 16 entre las escuelas registradas en el Ministerio de Educación (MINEDUC), totalizando $23(79,3 \%)$ de las 29 EDCP (año 2015).

Contenidos Encuesta: El cuestionario se basó en los dominios propuestos por la Asociación para la Educación Dental Europea (ADEE) (9) y aplicados por la Alianza para el Futuro Libre de Caries (ACFF) ${ }^{(16)}$ y la Asociación Colombiana de Facultades de Odontología (ACFO) ${ }^{(17)}$.

La construcción del cuestionario incluyó los cinco dominios definidos previamente en el documento de Consenso del Currículo de Cariología Europeo publicado por Shulte y cols. ${ }^{(8-9)}$ y posteriormente traducido al idioma español por Martignon y cols. ${ }^{(16)}$, en el que se agrega el dominio

\section{Salud Pública}

Los 6 dominios considerados en el cuestionario(9,16,17), (Tabla 1) y los 43 contenidos incluyeron preguntas sobre las metodologías educativas.

El cuestionario consideró 55 preguntas, de ellas, 43 sobre los contenidos impartidos y 12 preguntas destinadas a profundizar los contenidos de los dominios II, III y IV.

El cuestionario fue sometido al análisis y evaluación previa de contenido y lenguaje por un comité de expertos, formado por tres profesores (Cariología, Educación y Salud pública).

El cuestionario fue enviado por correo electrónico a los profesores encargados de las asignaturas de cariología cuando estos estaban identificados en el plan de estudios (publicados en las respectivas páginas

Tabla 1: Dominios del currículo de cariología según Shulte y cols. ${ }^{(11)}$ más el Dominio Salud Pública.

\begin{tabular}{l|l}
\hline Dominio I & Conocimiento base \\
\hline Dominio II & Valoración de riesgo, diagnóstico y síntesis \\
\hline Dominio III & Estrategias y terapia no quirúrgica \\
\hline Dominio IV & Decisiones de tratamiento operatorio \\
\hline Dominio V & Salud Pública \\
\hline Dominio VI & Cariología basada en la evidencia \\
\hline
\end{tabular}

web institucionales) o al director académico de la escuela, o secretario de estudios cuando correspondía, solicitándole expresamente a todos los encuestados, que aplicaran la encuesta incluyendo la recolección de información relacionada con la aplicación clínica de los contenidos. El cuestionario fue acompañado de una carta introductoria, donde se explicaban brevemente los objetivos del estudio, informando acerca de la codificación de las encuestas como método para el aseguramiento de la confidencialidad.

Recolección datos: El contacto con las EDCP, sus profesores, despacho, seguimiento y recepción de los cuestionarios, fue realizado por el coordinador del estudio. Este proceso se extendió por el período de tres meses (año 2015).

Análisis de los datos: Para el análisis ciego de los datos, los cuestionarios fueron codificados con números, el único investigador que conoció el origen del cuestionario fue el coordinador del estudio.

La data fue transferida a planilla Excel (Microsoft 2013) y procesada para su análisis estadístico descriptivo, por medio del programa estadístico Stata, versión 14.2. (StataCorp, Texas, USA).

\section{RESULTADOS}

La tasa de participación en el estudio fue de $86,95 \%$, (20 de 23 escuelas). El 100\% de los encuestados respondieron la totalidad de las preguntas.

Los resultados del resumen de los contenidos educativos impartidos, separados por dominio, modalidad educativa empleada y su respectiva aplicación clínica, expresados en porcentajes (Tabla 2).

Las respuestas de los cuestionarios en detalle, separados por dominio, contenido, metodología educativa empleada y aplicación clínica de los contenidos teóricos durante el proceso formativo, expresados en porcentaje (Tabla 3).

Tabla 2: Resultados separados por Dominio, expresados en porcentaje de los contenidos educativos impartidos, modalidad educativa empleada y su respectiva aplicación clínica.

Dominio I Conocimiento base

Dominio II Valoración de riesgo, diagnóstico y síntesis

Dominio III Estrategias y terapia no quirúrgica

Dominio IV Decisiones de tratamiento operatorio

Dominio V Salud Pública

Dominio VI Cariología basada en la evidencia

Media
Imparte Contenido

$71 \%$

$90 \%$

$65 \%$

$66 \%$

$75 \%$

$45 \%$

$69 \%$

$70 \%$

\section{Clase Teórica}

$69 \%$

$85 \%$

$60 \%$

$53 \%$

$60 \%$

$25 \%$

$59 \%$

$65 \%$
Paso Práctico

$12 \%$

$31 \%$

$10 \%$

$13 \%$

$20 \%$

$5 \%$

$15 \%$

$14 \%$
Aplicación Clínica $19 \%$

$36 \%$

$27 \%$

$26 \%$

$35 \%$

$0 \%$

$24 \%$

Promedio Ponderado por cantidad preguntas de los dominios 
Tabla 3: Resultados según dominio, contenidos, metodología educativa utilizada y su aplicación clínica declarada, expresados en porcentaje.

Dominio I. (17 preguntas)

Contenidos

Rol del biofilm en el proceso de caries

Teoría ecológica de Marsh

Rol del S. Mutans como principal agente etiológico

Etiología polimicrobiana de la caries

La teoría de caries de Fejerskov modificada

Conceptos de enfermedad / proceso y lesión de caries

La caries como enfermedad transmisible

La caries como enfermedad infecto contagiosa

El rol de la saliva en el proceso caries

Rol de la dieta en el proceso caries

Bioquímica de los tejidos duros del diente

Embriología e histología de tejidos dentarios duros

Apariencia clínica e histológica de la Caries de esmalte y dentina

Epidemiología de la caries

Índice significante de caries (SIC)

Etiopatogenia de las lesiones no cariosas( LNC)

Rol de la dieta en lesiones no cariosas

Dominio II. (5 preguntas)

Indicadores de riesgo cariogénico y predictibilidad

Medición del flujo salival

Conceptos y criterios de diagnóstico y detección de caries

Evaluación radiográfica de lesiones de caries

Caries radicular, etiopatogenia diagnóstico y tratamiento

Dominio III. (11 preguntas)

Análisis de las decisiones de tratamiento en caries y lesiones no cariosas

Control químico de la infección bucal

Uso de la clorhexidina para control de caries y/ S. mutans

Control mecánico del biofilm

Flúorterapia según riesgo cariogénico

Agentes remineralizantes no flúor

Uso de sellantes terapéuticos

Uso de sellantes proximales en lesiones no cavitadas

Uso de infiltrantes en lesiones proximales no cavitadas

Fundamentos del uso de Probióticos en el control de caries

Fundamentos del uso de Prebióticos en el control de caries

Dominio IV. (8 preguntas )

Tratamiento de caries dentinaria profunda

Técnica ART

Evaluación y tratamiento de caries secundaria, recurrente

El Rol de las Metaloproteinasas en la progresión de caries dentinaria

Análisis crítico de los principios cavitarios de Black

Fundamentos de odontología de mínima intervención

Diseño de preparaciones cavitarias biológicas

Instrumental rotatorio y manual para eliminación de dentina y

preparación cavitaria

Dominio V. (1 pregunta)

Promoción y prevención de la salud

Dominio VI. (1 pregunta)

Análisis crítico de la evidencias

\begin{tabular}{|c|c|c|c|}
\hline $\begin{array}{c}\text { Imparte } \\
\text { Contenido } \\
(\%)\end{array}$ & $\begin{array}{c}\text { Clase } \\
\text { Teórica } \\
(\%)\end{array}$ & $\begin{array}{c}\text { Paso } \\
\text { Práctico } \\
(\%)\end{array}$ & $\begin{array}{l}\text { Aplicación } \\
\text { Clínica } \\
(\%)\end{array}$ \\
\hline 100 & 100 & 30 & 35 \\
\hline 90 & 85 & 15 & 30 \\
\hline 55 & 60 & 10 & 15 \\
\hline 80 & 70 & 10 & 0 \\
\hline 95 & 95 & 15 & 20 \\
\hline 30 & 35 & 0 & 5 \\
\hline 30 & 35 & 0 & 5 \\
\hline 35 & 40 & 0 & 5 \\
\hline 100 & 100 & 30 & 35 \\
\hline 100 & 100 & 25 & 50 \\
\hline 90 & 90 & 10 & 15 \\
\hline 60 & 55 & 20 & 10 \\
\hline 100 & 90 & 30 & 25 \\
\hline 80 & 70 & 0 & 15 \\
\hline 45 & 35 & 5 & 0 \\
\hline 60 & 60 & 5 & 25 \\
\hline 60 & 60 & 5 & 30 \\
\hline 100 & 100 & 35 & 40 \\
\hline 95 & 90 & 35 & 40 \\
\hline 100 & 95 & 55 & 40 \\
\hline 90 & 80 & 25 & 45 \\
\hline 65 & 60 & 5 & 15 \\
\hline 75 & 75 & 20 & 30 \\
\hline 55 & 50 & 5 & 30 \\
\hline 65 & 60 & 0 & 30 \\
\hline 90 & 85 & 20 & 45 \\
\hline 90 & 90 & 15 & 50 \\
\hline 65 & 55 & 0 & 20 \\
\hline 90 & 80 & 25 & 45 \\
\hline 75 & 65 & 10 & 40 \\
\hline 65 & 60 & 10 & 10 \\
\hline 45 & 35 & 0 & 0 \\
\hline 5 & 5 & 0 & 0 \\
\hline 85 & 70 & 15 & 45 \\
\hline 85 & 65 & 15 & 40 \\
\hline 45 & 30 & 5 & 30 \\
\hline 75 & 65 & 0 & 5 \\
\hline 35 & 30 & 10 & 5 \\
\hline 95 & 90 & 20 & 35 \\
\hline 50 & 35 & 15 & 20 \\
\hline 60 & 40 & 25 & 30 \\
\hline 75 & 60 & 20 & 35 \\
\hline 45 & 25 & 5 & 0 \\
\hline
\end{tabular}

Las respuestas que profundizan los contenidos teóricos impartidos por las EDCP proporcionaron información sobre los contenidos enseñados teóricamente y aplicados por los alumnos en su práctica clínica, expresados en porcentaje (Tabla 4).

\section{DISCUSIÓN}

Este es el primer estudio que explora cuantitativamente los contenidos impartidos en cariología por las EDCP, permitiendo visualizar como los contenidos teóricos se aplican en la práctica clínica y es el segundo cuestionario que se realiza en el país. La primera tuvo como objetivo describir el estado actual de la enseñanza de Cariología, detectando que la mitad de las universidades chilenas considera que la cariología no está implementada apropiadamente, especialmente por la mezcla de conceptos tradicionales y modernos ${ }^{(14)}$

El presente estudio tuvo alta tasa de respuesta $(86,95 \%)$ similar a otros estudios que utilizaron correo electrónico, como el estudio en 
Tabla 4: Profundización de los contenidos enseñados teóricamente y aplicados por los alumnos en su práctica clínica, expresados en porcentaje.

\section{Contenido}

Método(s) de detección de caries

\begin{tabular}{|c|c|c|}
\hline examen visual & $95 \%$ & $65 \%$ \\
\hline visual tactil sonda aguzada & & $5 \%$ \\
\hline Visual y táctil con sonda OMS & $65 \%$ & $5 \%$ \\
\hline el examen radiográfico complementario Bite Wing. & $65 \%$ & $55 \%$ \\
\hline imparte métodos emergentes de detección & $57 \%$ & $5 \%$ \\
\hline \multicolumn{3}{|l|}{ Criterios de detección de lesiones de caries } \\
\hline criterio OMS & $35,70 \%$ & $25 \%$ \\
\hline criterio ICDAS & $35,70 \%$ & $40 \%$ \\
\hline criterio Nyvad & $21,30 \%$ & $20 \%$ \\
\hline criterio Univiss & $7,10 \%$ & \\
\hline no responde o no sabe & & $10 \%$ \\
\hline \multicolumn{3}{|l|}{ Herramienta de evaluación de riesgo cariogénico en niños } \\
\hline Cariogram & $85 \%$ & $50 \%$ \\
\hline CAMBRA & $10 \%$ & $30 \%$ \\
\hline CAST & & $5 \%$ \\
\hline no sabe o no responde & $5 \%$ & $30 \%$ \\
\hline \multicolumn{3}{|l|}{ Herramienta de evaluación de riesgo cariogénico en adultos } \\
\hline Cariogram & $75 \%$ & $65 \%$ \\
\hline CAMBRA & & $25 \%$ \\
\hline \multicolumn{3}{|l|}{ CAST } \\
\hline no sabe o no responde & $25 \%$ & $30 \%$ \\
\hline \multicolumn{3}{|l|}{ Estrategias de tratamiento de lesiones de caries en niños } \\
\hline Tratamiento no invasivo & $95 \%$ & $70 \%$ \\
\hline Intervención mínimamente invasiva & $95 \%$ & $80 \%$ \\
\hline tratamiento quirúrgico o convencional & $95 \%$ & $65 \%$ \\
\hline no sabe o no responde & $5 \%$ & $15 \%$ \\
\hline \multicolumn{3}{|l|}{ Estrategias de tratamiento de lesiones de caries en adultos } \\
\hline Tratamiento no invasivo & $80 \%$ & $55 \%$ \\
\hline Intervención mínimamente invasiva & $88 \%$ & $70 \%$ \\
\hline tratamiento quirúrgico o convencional & $100 \%$ & $60 \%$ \\
\hline \multicolumn{3}{|l|}{ no sabe o no responde } \\
\hline \multicolumn{3}{|l|}{ Técnica de tratamiento de caries dentinaria profunda en niños } \\
\hline remoción total del tejido dentinario infectado y afectado & $25 \%$ & $25 \%$ \\
\hline remoción parcial escalonada (Step Wise) & $60 \%$ & $75 \%$ \\
\hline Remoción Ultraconservadora & $20 \%$ & $45 \%$ \\
\hline Pulpotomía preventiva & $20 \%$ & $25 \%$ \\
\hline Técnica Hall & $15 \%$ & $10 \%$ \\
\hline no sabe o no responde & $10 \%$ & $20 \%$ \\
\hline \multicolumn{3}{|c|}{ Técnica de tratamiento de caries dentinaria profunda en adultos } \\
\hline remoción total del tejido dentinario infectado y afectado & $25 \%$ & $30 \%$ \\
\hline remoción parcial escalonada (Step Wise) & $65 \%$ & $70 \%$ \\
\hline Remoción Ultraconservadora & $20 \%$ & $9 \%$ \\
\hline Pulpotomía preventiva & $5 \%$ & \\
\hline Técnica Hall & $5 \%$ & \\
\hline no sabe o no responde & $10 \%$ & $30 \%$ \\
\hline \multicolumn{3}{|l|}{ Índices de detección y valoración de las lesiones no cariosas } \\
\hline BEWE (Basic Erosive Wear Examination) & $20 \%$ & $15 \%$ \\
\hline Smith and Knight & & $5 \%$ \\
\hline ninguno & $60 \%$ & $55 \%$ \\
\hline no sabe o no responde & $20 \%$ & $30 \%$ \\
\hline
\end{tabular}

Estados Unidos por Fontana y cols. ${ }^{(18)}(83 \%)$, y Diaz-Yokens y cols. ${ }^{(17)}$ en Chile $(85,7 \%)$ en comparación con aquellos con menor tasa de respuesta como Clark y Mjör ${ }^{(12)}$ en EEUU (66\%), el realizado en Europa por Schulte y cols. ${ }^{(9)}(72 \%)$ y el efectuado en EEUU. por Funkhouser y cols. ${ }^{(19)}(76 \%)$. La diferencia entre los estudios que usaron el correo electrónico, se debe probablemente, a que en el presente se definió el rol de un coordinador, que realizó el seguimiento a los encuestados durante el estudio.

Analizado el conjunto de dominios, se observó alto promedio ponderado

Enseñados teoricamente

Utilizados en la práctica clínica

$85 \%$

Visual y táctil con sonda OMS

de contenidos impartidos (70\%), entregado mayoritariamente por medio de clases teóricas $(65 \%)$, que contrasta con la baja utilización de las prácticas de laboratorio (14\%) junto al bajo porcentaje de contenidos aplicados en la clínica (24\%). Si a este hecho se suma, el bajo porcentaje de respuestas afirmativas (45\%) en el dominio VI, junto con que estos se imparten en clases teóricas $(24 \%)$ y no consideran metodologías educativas prácticas $(0 \%)$, ni aplicación clínica $(0 \%)$, esta situación podría condicionar negativamente el comportamiento preventivo/curativo del futuro profesional, frente al manejo 
de la enfermedad. Situación parecida fue observada por Martignon y cols. ${ }^{(13)}$ en Latinoamérica, donde el $77,8 \%$ de los estudiantes participaron en talleres de práctica preclínica, con baja transferencia a la práctica clínica $(48 \%)$. Esto contrasta con lo reportado por Schulte y cols..$^{(8)}$ cuando señala que la educación teórica en cariología en Europa, es entregada por prácticamente todas las escuelas europeas y los ejercicios preclínicos son ofrecidos por el $98 \%$ de las escuelas. Recientemente, la experiencia en Canadá muestra que realizan laboratorios prácticos para estudiantes de pregrado en cariología previo al ingreso a la clínica en el $60 \%$ de las escuelas, superior al $14 \%$ encontrado en Chile. Al igual que en el presente estudio, en las escuelas canadienses se observó que todas ellas carecían de la adecuada integración de la educación en cariología durante la capacitación clínica. (20) Por otra parte Afrashtehfar y cols. ${ }^{(21)}$ y Graham cols. ${ }^{(22)}$ demuestran, que los conocimientos deben necesariamente traducirse en acciones, en este sentido, otro hecho relevante en educación odontológica, es el destacado por Werb y Matea ${ }^{(23)}$, quienes afirman que la falta de capacitación, apoyo y conocimiento metodológico en educadores odontológicos, son una barrera, ya que la educación debe proporcionar a los futuros profesionales las herramientas necesarias para adaptarse constantemente a los avances.

El dominio II fue el de mejor comportamiento del estudio, observándose la mayor respuesta sobre contenidos impartidos $(90 \%)$, que se asocia con la más alta tasa de aplicación clínica de contenidos (36\%) y modalidad educativa práctica de mayor entrenamiento por los estudiantes, sin embargo, también presenta gran asimetría de respuestas al comparar los instrumentos educativos teóricos $(85 \%)$ y prácticos declarados $(31 \%)$. Al contrastar los resultados de la profundización de los contenidos (tabla 4) con los obtenidos en el estudio canadiense ${ }^{(20)}$, el dominio II presentó semejanzas en la enseñanza de los métodos de detección de caries: visual $90 \%$ versus $95 \%$ de las escuelas chilenas; táctil $90 \%$ que es superior al $65 \%$ observado en Chile; mientras en el criterio de detección ICDAS, es mayoritariamente enseñado en Canadá (60\%), versus 36\% en Chile; lo mismo acontece en la evaluación de actividad de caries $90 \%$ en Canadá versus $21,3 \%$ en Chile. En relación la enseñanza del examen radiográfico complementario, también se observa diferencias, mientras en Canadá alcanza $100 \%$, en Chile fue del $65 \%{ }^{(20)}$

El segundo dominio de mejor implementación en cariología es el $\mathrm{V}$, con alto nivel de contenidos impartidos $(75 \%)$, con entrega de clases teóricas $(60 \%)$, bajo entrenamiento práctico en laboratorio $(20 \%)$ y parcial aplicación clínica (35\%), especialmente mayor que en los otros dominios con excepción del dominio II.

Al analizar las respuestas de los contenidos impartidos en el Dominio I, se observa consenso en la entrega del contenido "rol del biofilm en el proceso de caries" (100\%) y simultáneamente bajo promedio en la entrega del contenido "Concepto de Caries Enfermedad / Proceso y Lesión" (30\%). La entrega de contenidos, tales como: "la caries como enfermedad transmisible" (30\%) y "caries como enfermedad infectocontagiosa" (30\%), conceptos actualmente superados como explicación etiopatogénica ${ }^{(24)}$ aún mantienen su presencia en el currículo, lo que se interpreta como desactualización de contenidos. Por otra parte, en relación a: "El rol de la saliva en el proceso de caries" y el "Rol de la dieta en el proceso de caries " que se entregan en el $100 \%$ de las escuelas dentales en forma teórica, son temas de muy baja inclusión en pasos prácticos y aplicación clínica $30 \%-25 \%$ y $35 \%-50 \%$ respectivamente, Estos resultados del Dominio I se aprecian alineados con los observados en el Dominio II, donde los contenidos "Indicadores de riesgo cariogénico y predictibilidad" y "Medición del flujo salival" presentan niveles de aplicación clínica de solo $40 \%$. En general en las respuestas del Dominio II se repite el bajo uso de pasos prácticos y aplicación clínica de los contenidos, hecho complejo ya que las 5 preguntas de este dominio son de gran relevancia clínica, situación que se observa de mayor gravedad cuando se analiza el contenido "Caries radicular, etiopatogenia, diagnóstico y tratamiento", con extrema reducción de aplicación clínica (15\%), hecho relevante si se tienen a la vista los datos demográficos de la población, que señalan que las proyecciones para final de este decenio, en el segmento etario tercera edad, constituirá el $15 \%$ del total de los habitantes.

Otro contenido esencial para el ejercicio profesional del Dominio I es la identificación de la "Apariencia clínica e histológica de la Caries de esmalte y dentina", que permite reconocer características visuales y táctiles del diente enfermo, cuyo entrenamiento está destinado a adquirir competencias de diferenciación de los tejidos sanos de enfermos e identificar y discriminar otras afecciones de los tejidos dentarios (erosiones, hipoplasias, crack, sensibilidad dentaria), este contenido se imparte en el $100 \%$ de las escuelas, con alta metodología teórica, reducida actividad de laboratorio (30\%) y escasa aplicación clínica $(25 \%)$.

De igual forma el Dominio III mantiene la tendencia de baja aplicación clínica en contenidos tan relevante como "control mecánico del biofilm" (45\%),"fluorterapia según riesgo cariogénico" (50\%) y "uso de sellantes terapéuticos"(45\%), (el resultado ideal sería cercano al 100\%), hecho de gran preocupación porque implica entrenamiento preventivo en el proceso formativo, con el objeto de continuar el aumento de pacientes libres de lesiones de caries durante los próximos años, según lo declarado tanto por los objetivos sanitarios nacionales como por la Organización Mundial de la Salud (OMS). ${ }^{(2)}$ En el estudio de Martignon y cols. ${ }^{(13)}$ se observa similar situación, donde reportaron que sus escuelas enseñaban estrategias preventivas pero solo el $43.4 \%$ lo trasladaba a evaluación de riesgo y el $40.7 \%$ respondió que implementan el manejo no quirúrgico regularmente. Por otra parte, si se analizan los resultados de las estrategias aplicadas en la práctica clínica para tratamiento de lesiones de caries en niños y adultos, se observan diferencias de contenidos al enseñar tratamientos no invasivos en niños $(70 \%)$, versus adultos (55\%), efectuado el cruce de resultados, como herramienta de verificación, confirma las tendencias observadas en el análisis del Dominio III.

En el dominio IV, se observa que los contenidos: "Análisis crítico de los principios de Black" y "Diseño de preparaciones cavitarias biológicas", presentan bajo porcentaje de respuestas afirmativas $35 \%$ y $50 \%$ ), acompañado de bajos porcentajes de utilización de metodologías educativas, como clases teóricas (30\% y $35 \%$ ) y menores aún en pasos prácticos $(10 \%$ y $15 \%)$ y aplicación clínica $(5 \%$ y $20 \%)$, una explicación posible a este resultado, podría buscarse en la posibilidad que estos contenidos son impartidos por otras asignaturas (10 y $15 \%$ ) o no se imparten.

La mayoría $(75 \%)$ de las EDCP define la asignatura de Cariología como responsable de la enseñanza de la etiopatogenia de la caries y lesiones no cariosas, el $10 \%$ en Odontopediatría y el $5 \%$ en Operatoria Dental, además, el $60 \%$ de los contenidos de Cariología se imparten en la asignatura o curso de Cariología y el $40 \%$ se enseñan de forma integrada a otras asignaturas. También cabe destacar que la mayoría de los contenidos de cariología $(65 \%)$, se encuentran programados como curso o asignatura semestral y solo el 15\% (3 escuelas), lo imparten anualmente, la ubicación en la malla curricular de la asignatura es entre el $5^{\circ}$ semestre $(45 \%)$ y el $6^{\circ}$ semestre $(25 \%)$ de la carrera. Otro dato relevante, es el número de académicos que participan en forma estable la asignatura de Cariología, fluctúa entre 3 a 4 en 8 escuelas (42\%), 2 docentes en 3 escuelas (15\%) y más de 4 en 4 escuelas (21\%).

La posible explicación del escaso uso de prácticas de laboratorio podría relacionarse con la baja cantidad de horas destinadas en el currículo a estos contenidos, hecho aún no determinado y que constituye un nuevo campo de investigación, sin embargo, podría relacionarse con el mayor costo que significa la implementación de actividades prácticas, donde la relación número de estudiante por académico debe ser menor, además de requerir espacios físicos y recursos para pasos prácticos o de laboratorio, no obstante, ninguno de estos argumentos resultan válidos frente a la enorme prevalencia de la enfermedad(6) y la existencia de evidencia teórica y práctica, que permitirían a la gran mayoría de la población ubicarse en el segmento libre de la enfermedad, con la correcta aplicación de medidas preventivas y de control de la enfermedad(25).

Los dominios III (estrategias y terapias no quirúrgicas) y IV (decisiones de tratamiento operatorio) muestran similar nivel de contenidos impartidos (65\% y $66 \%$ ) y de aplicación clínica ( $27 \%$ y $26 \%$ ), ambos porcentajes son bajos para la importancia clínica del dominio y reflejan el establecimiento de contenidos de terapias no quirúrgicas para el manejo de lesiones cariosas al mismo nivel que métodos operatorios, que comparado con el estudio previo debe considerarse como un avance, dado que Diaz-Yokens y cols. ${ }^{(14)}$ observaron baja adopción de filosofía de tratamiento no invasiva, (manejo no quirúrgico), no encontrándose adecuadamente implementado en los planes de estudio.

Finalmente, a pesar de la diferente cantidad de preguntas efectuadas por dominio, el análisis de los promedios aritmético y promedio ponderado por la cantidad de preguntas, refleja franca similitud de resultados, hecho que podría expresar que la enseñanza de los contenidos educativos en cariología, es un solo proceso continuo, donde las dificultades de desarrollo de algunos dominios, se reflejan a lo largo de todo el proceso educativo y no necesariamente afectan un dominio específico del saber.

\section{CONCLUSIÓN}

La aplicación del cuestionario sobre el estado actual de los contenidos de cariología impartidos por las escuelas dentales chilenas, muestra enseñanza con gran cantidad de contenidos, preferentemente utilizando clase teórica como metodología educativa, que en su mayoría no se traducen en entrenamiento práctico de laboratorio y presentan escasa aplicación clínica de los contenidos desarrollados.

\section{CONFLICTO DE INTERÉS}

Los autores declaran que no tienen ningún conflicto de interés. 


\section{Bibliografía}

1. Ismail Al, Tellez M, Pitts NB, Ekstrand KR, Ricketts D, Longbottom C, et al. Caries management pathways preserve dental tissues and promote oral health. Community Dent Oral Epidemiol. 2013;41(1):e12-40.

2. Petersen PE, Bourgeois $\mathrm{D}$, Ogawa $\mathrm{H}$, et al. The global burden of oral diseases and risks to oral health. Bull World Health Organ 2005; 83(9):661-9.

3. Akesson ML, Wärnberg Gerdin E, Söderström U, Lindahl B, Johansson I. Healthrelated quality of life and prospective caries development. BMC Oral Health. 2016;16:15.

4. Kassebaum N, Bernabé E, Dahiya M, Bhandari B, Murray CJ, Marcenes W. Global burden of untreated caries: a systematic review and metaregression. J Dent Res 2015;94(5):650-658.

5. Urzua I, Mendoza C, Arteaga O, Rodríguez G, Cabello R, Faleiros S, et al. Dental caries prevalence and tooth loss in Chilean adult population: first national dental examination survey. Int J Dent. 2012:2012:810170

6. Ministério da Saúde, Coordenação Nacional de Saúde Bucal. Condições se saúde bucal da população Brasileira 2002-2003 Resultados principais. Editora MS, 2005. (Consultado 30/07/2017). Disponible en http://bvsms.saude.gov.br/bvs/ publicacoes/condicoes_saude_bucal.pdf

7. Gerbert B, Badner V, Maguire B, Martinhoff J, Wycoff S, Crawford W. Recent graduates' evaluation of their dental school education. J Dent Educ. 1987;51:697700.

8. Schulte AG, Buchalla W, Huysmans MC, Amaechi BT, Sampaio F, Vougiouklakis G. et al. A survey on education in cariology for undergraduate dental students in Europe. Eur J Dent Educ. 2011;15(Suppl 1):3-8.

9. Schulte AG, Ptts B, Huysmans MC, et al. European core curriculum in cariology for undergraduate dental students. Caries Res 2011;45:336-345.

10. Fukushima M, Iwaku M, Mjor IA. Cariology in Japanese dental schools. Int Dent J 2004;54:269-272.

11. Fontana M, Horlak D, Sharples S. Teaching of cariology in U.S. dental schools J Dent Res 2012; 91(Spec Iss A)Abstract 313.

12. Clarck TD, Mjor IA. Current teaching of cariology in North American dental schools. Oper Dent 2001;26:412-418.

13. Martignon S, Gomez J, Tellez M, Ruiz JA, Marin LM, Rangel MC. Current cariology education in dental schools in Spanish-speaking Latin American countries. J Dent Educ. 2013;77(10):1330-7.

14. Diaz-Yokens M, González S, Giacaman R, Araya-Bustos M, Moncada G, Martignon S. Cariology curriculum in Chilean universities. Rev Clin Periodoncia Implantol Rehabil Oral. 2018;11(2):98-101.
15. Nascimento MM, Behar-Horenstein LS, Feng X, Guzmán-Armstrong S, Fontana M. Exploring how U.S. dental schools teach removal of carious tissues during cavity preparations. J Dent Educ. 2017;81(1):5-13.

16. Martignon S, Marín LM, Pitts N, Jácome-Liévano S. Consensus on domains, formation objectives and contents in cariology for undergraduate dental students in Colombia. Eur J Dent Educ. 2014;18(4):222-33.

17. Martignon S, Liévano SJ, Marín LM. Consenso sobre dominios, objetivos de formación y contenidos de cariología para pregrado de odontología. Alianza por un futuro libre de caries. Capítulo Colombia, Frente Académico. 2013. (Consultado 30/07/2017). Disponible en http://www.alianzaporunfuturolibredecaries.org/ Caries/Tools/es/co/colombia-chapter/ConsensoEnsenanzaenCariologia-ACFFCOLOMBIA.pdf .

18. Fontana M, Guzmán-Armstrong $S$, Schenkel $A B$, Allen $K L$, Featherstone J, Goolsby S, et al. Development of a core curriculum framework in cariology for U.S. dental schools. J Dent Educ. 2016;80(6):705-20.

19. Funkhouser E, Fellows JL, Gordan VV, Rindal DB, Foy PJ, Gilbert GH. Supplementing online surveys with a mailed option to reduce bias and improve response rate: the national dental practice-based research network. J Public Health Dent. 2014; 74(4):276-82

20. Tikhonova S, Girard F, Fontana M. Cariology education in Canadian dental schools: Where are we? Where do we need to go? J Dent Educ. 2018;82(1):39-46. 21. Afrashtehfar KI, Assery MK. From dental science to clinical practice: knowledge translation and evidence-based dentistry principles. Saudi Dent J. 2017;29(3):83-92. 22. Graham ID, Logan J, Harrison MB, Straus SE, Tetroe J, Caswell W, et al. Lost in knowledge translation: time for a map? J Contin Educ Health Prof. 2006;26(1), 13-24.

23. Werb SB, Matear DW. Implementing evidence-based practice in undergraduate teaching clinics: a systematic review and recommendations. J Dent Educ. 2004;68(9):995-1003.

24. Simon-Soro A, Mira A. Solving the etiology of dental caries. Trends Microbiol. 2015; 23(2):76-82.

25. Baiju RM, Peter E, Varghese NO, Sivaram Rj, Oral heath and quality of life: current concepts. Clin Diagn Res. 2017;11(6):ZE21,ZE26. 